\title{
Knowing versus understanding: adjusting the contextual lens in safety science
}

\author{
Janice $R$. McCall ${ }^{1, *}$ \\ ${ }^{1}$ Saint Louis University, Parks College of Engineering, Aviation and Technology, United States of \\ America
}

\begin{abstract}
Preparing for the unknown safety threat requires new ways of studying safety in today's highly complex and dynamic environment. Qualitative inquiry offers advantages to expand the contextual lens in safety science by broadening understanding of the environment and conditions people negotiate in normal day-to-day operations. Through the narrative accounts of those with first-hand experience qualitative researchers seek to narrow the gap between work-as-done and work-as-imagined. It is through their individual experience, when analyzed collectively, that a greater context emerges. To aid in determining which research method is best for investigating safety phenomenon, this paper offers an overview of the philosophical underpinnings of qualitative research as well as recent examples from aviation, maritime, and mining.
\end{abstract}

\section{Introduction}

In the study of safety, knowing what happened is different than understanding how and why an event occurred. It is here, in the space between knowing and understanding, that interpretive qualitative research contributes a great deal to safety science. Safety science "refers both to what we know about safety and to the ways we have built and continue to build this knowledge" [1]. While qualitative, quantitative, and critical realism all enhance safety research, qualitative research's diversity of ontology, epistemology, and methodology, is well situated to expand context, foster understanding, and strengthening organizational safety learning and resilience. In the complex and dynamic environment, where human life hangs in the balance and safety precariously exists, "sometimes your greatest strength can emerge as a weakness if the context changes" [2].

Of the three research methodologies common in safety science, quantitative, qualitative, and more recently critical realism; quantitative research has dominated. The quantitative approach "has been, and continues to be, the preferred research methodology" [3]. This preference is often driven by the relationship between quantitative research and business management. The management-measurement arrangement capitalizes on the strengths of quantitative research allowing for relatively fast, precise, unbiased, statistical analysis that can determine trends and be generalized to a larger population.

For comparison, a recent quantitative study of seven European airlines, by Kaspers, Karanikas, Piric, van Aalst, de Boer, \& Roelen [4] concluded "that there is limited empirical

*Corresponding author: jan.mccall@slu.edu 
evidence about the relationship between Safety Management System (SMS) processes and safety outcomes." Yet, these finding lack the detail from accounts of those working with SMS. Similar findings in a Størkersen, Antonsen, and Kongsvik, [5] qualitative study of Norwegian maritime passenger transportation reported that while personal injury had decreased, "ship accidents had increased in the period during which safety management has been regulated." Interviews conducted with 47 regulators, managers, and crewmembers about their experience with SMS revealed negative consequences to navigation such as "unthinking compliance" and that the "administrative burden is easily associated with less concentration on navigation" [5]. Qualitative inquiry captures a glimpse of the "stocks of images in our heads, put there by our origins, upbringing and experience of the world, which we use to make sense of the world" [6].

These two studies reveal a weakness in quantitative research, that is a strength of qualitative. The deductive approach "fails to provide insight into the participants' individual or personal experiences. They do not let the respondents describe their feelings, thoughts, frames of reference, and experiences with their own words" [7]. Qualitative research weaknesses include being time consuming, biased, and seldom generalizable.

These strengths and weaknesses led to the early association of quantitative as hard, fixed, and objective while qualitative was considered soft, flexible, and subjective [8]. Hong and Lang [9] apply the Chinese philosophy Yin-Yang to these two methodologies, where power "comes from capturing the very nature of the world and the mechanism through which the world operates." This is where the overreliance on quantitative research limits our ability to capture the human experience, the messy details, that create the context surrounding safety.

\subsection{Purpose}

Rather than exam all three methodologies, quantitative, qualitative, and critical theory, this paper attempts to engage the reader in a deeper understanding of the benefits and applications of qualitative research in safety. Safety practitioners, mangers, and anyone interested in the human experience may find this paper worthwhile. It is divided into sections addressing how qualitative research uses individually constructed experience, collectively analyzed, to illustrate context in order to broaden understanding of safety issues. The sections include, the meaning of context as used in qualitative research, the role context plays in knowing and understanding, and a brief review of the philosophical underpinnings of interpretive qualitative. The final section offers advantages of qualitative research and highlights its usefulness in three recent safety studies.

\section{Context}

"If an intervention is a seed then the context is the soil" [10].

Context is a complicated, commonly used term, that carries a wide range of meanings. Rather than delve into the multitude of definitions of context, including 150 analyzed by Bazire and Brézillon [11], covering "various disciplines such as computer science, philosophy, economy, business, HCI [Human Computer Interface]etc." this paper focuses on context as derived from lived experience through qualitative research in safety. Van Dijk [12] argued that "the notion of context as it is used in the social sciences is not a strictly theoretical concept, but rather a more or less fuzzy notion denoting a situational, historical, geographical, social or cultural environment of a phenomenon being studied." Context, in this regard, aligns 
with social sciences, sociology, and linguistics, by focusing on gaining meaning and a broader understanding of events, including organizational safety conditions and culture.

Safety cannot be understood and explained at a distance from the cultural and social environment in which it appears. To the contrary, safety, like any organizational endeavor, requires a balance between practice (work-as-done), and process (work-as-imagined). Closing the space between the two entails what Brown and Duguid [13] refer to as "collective wisdom," putting individual stories together and drawing from the experience of a group.

Described through qualitative study of lived experiences, we see context returned to its early Latin origins contextus meaning "a joining together," or contexere "to weave together" [14] where multiple narratives join to create context in safety. The qualitative researcher is not looking for the explanation of a safety event, or the root cause, rather an interpretation of how each person involved experienced the event, and a collective context from which to garner understanding of the organizational safety conditions, culture, and systems in a holistic manner [15].

Each description of a safety event is shaped from the experience within the sociotechnical environment, then interpreted, and meaning constructed, within the minds of the individuals. Technology cannot replace experience, values, and judgement [16]. It is the lived experience and judgment found in capturing and analyzing that yields a broader context. "We collect safety stories because stories provide context. Stories we hear are what storytellers choose to tell because it is important to them. We often learn about safety issues that management or expert consultants had no clue about" [17]. To borrow from Duranti and Goodwin's [18] study of linguistics, what the qualitative researcher "seeks to describe is not what they consider context, for example their map of the city in which the blind man finds himself, but rather how the subject himself attends to and organizes his perceptions of the events and situations he is navigating through."

Such views diverge from traditional positivist interpretations of context, but when used concurrently, offer new insights into safety through organizational systems functions and the environment employees negotiate in day-to-day operations to ensure safety. In the "positivist, rational-analytic philosophy that has always dominated in science and medicine, context is usually defined as an 'objective' phenomenon, something 'real', something tangible and 'out there' - factors, variables, objects, events, domains and so on" [19]. There is risk in such belief that fails to see the human construct of context. Hummerdal [20] characterized this risk when he said; "our belief in best practice, standardized methods, and other versions of the one best way, can be seductive and create a blindness to the contextual adaptations that are required to make things work in everyday performance."

While the definition of context may end where it started, as a complicated, complex, and commonly-used term, its critical importance to advancing safety is clear; success in capturing context in safety is not found in the division of methods, but more accurately, when combined to provide greater understanding. Dixon-Woods [21] proposed focusing on "practical wisdom" and a blend of methods as the best way forward.

\subsection{Context in Knowledge and Understanding}

Before turning to philosophy, let us first explore how context, drawn from qualitative inquiry, contributes to knowledge and translates to understanding in safety science. Context provides familiarity and connects learning to meaning as part of knowledge development [22]. Safety is dependent on both individual and organizational learning [23-24]. "Cognitive theories tell us that learning occurs not by recording information but by interpreting it" [25]. This type of learning in safety involves gaining 'meaning' from knowledge. Safety learning, or any 
learning, is "knowledge-dependent; people use current knowledge to construct new knowledge" [25].

Knowledge, like context, is not easily defined. Nonetheless, in safety it may be useful to view knowledge as "an ordering and organization of a world constituted by our experience" [26]. Piaget's [27] theory of constructivism argued that "to know an object, to know an event, is not simply to look at it and make a mental copy or image of it." Piaget considered knowledge as acquired through cognitive schema that formed structures, categories, and transformed objects based on experience. Perhaps the easiest way to think of knowledge is to apply Perkins' [28] definition; "Knowledge is information on tap."

To expand insight into the safety environment, qualitative inquiry must consider the person(s) knowledge of events, what it meant individually and collectively, and how they understood the event to shape the collective context. Figure 1 portrays how individual context is translated into meaning, knowledge, and understanding, that when gathered and analyzed through qualitative inquiry presents a broader context for study. Each circle represents the individual's development of understanding through context, meaning, and knowledge within the web of safety culture. While each experience is valuable, it is the combined analysis that provides collective context for organizational safety learning and understanding.

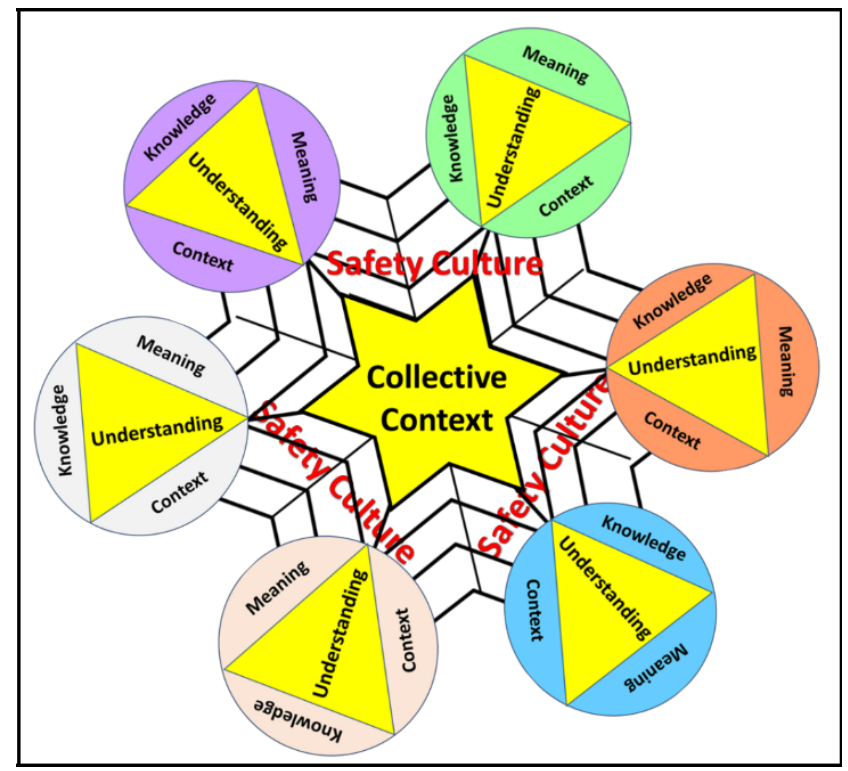

Figure. 1. Qualitative process of gathering and analyzing data, moving from individual context to meaning, knowledge, understanding, and collective context.

Understanding takes many forms in the qualitative research process. It begins with collection of individual data from the first-hand experience of those involved, in the form of interviews, observations, questionnaires, and narratives accounts. The analysis process then builds to a collective understanding through review of all those involved. During the analysis process, themes emerge that redefine and clarify context for further safety studies. Qualitative analysis is like putting together a jigsaw puzzle without the picture on the box. The qualitative researcher has a general idea there is something to be found within the data, based on the experience of those involved, but cannot interpret it without seeing how the pieces work together to form collective themes. Collective themes expand our understanding and reshape the contextual lens to identify where the gaps exist between work-as-imagined and word-asdone. 
Safety today is perhaps more challenging than ever, existing in complex and dynamic environments, that require new approaches. The ability to rely on past performance as an indicator of future success is limited; current safety theorists have turned to capacity, reliability, and resilience as key indicators of safety's future performance [29-32]. Knowing is not enough, we must expand our understanding in order to build flexibility and capacity into safety systems. It is time to revisit understanding as Perkins' defined it in 1998; "Understanding is the ability to think and act flexibly with what one knows." Understanding is a "flexible performance capability" [28] that allows individuals and organizations to adapt and quickly react to unexpected safety events.

In safety it is not always the unknown that presents the greatest threat, it is what is known and not shared. Often, these are the day-to-day adaptations we don't understand, that people make to ensure safety and efficiency, and which lie outside the very detailed and structured scope, Standard Operating Procedures (SOPs), checklists, policies, and regulations. "Work gets done because of people's effective informal understandings, their interpretations, their innovations and improvisations outside those rules" [33], which you will see in the case studies at the end.

This begs the question at the core of the Safety Differently and Safety II movement; If the same employees are doing the same work safely and successful $99.9 \%$ of the time, why is our attention focused on learning from the random and unpredictable failure? Hollnagel, Wears, \& Braithwaite [34] call for a new definition of safety gets to the heart of the importance of expanding understanding.

The definition of safety should be changed from 'avoiding that something goes wrong' to 'ensuring that everything goes right'. Safety-II is the system's ability to function as required under varying conditions, so that the number of intended and acceptable outcomes (in other words, everyday activities) is as high as possible. The basis for safety and safety management must therefore be an understanding of why things go right, which means an understanding of everyday activities [34].

By listening to workers unique experiences, we enter a perspective in which we see normal humans doing normal work while trying to create success amongst scarcity, imperfect and conflicting setups [35].

The goal of listening to and collecting employees' stories, as Conklin [36] asserts, is "to focus learning on the context before the consequence - the context is the only place you can have an impact on improving your organization." Moving away from safety outcomes as resultant and toward safety outcomes as emergent is one of the strengths of qualitative research.

\section{Philosophical Underpinnings of Qualitative Research}

To some, the philosophical underpinnings of qualitative research may seem unimportant in the study of safety, burdensome, and dated. Nonetheless, there are philosophical differences of critical importance to safety. Highlighted below are certain distinctions in research paradigms along with selected preeminent philosophical foundations guiding safety science.

The situated researcher approaches the world with a set of ideas, a framework (theory, ontology) that specifies a set of questions (epistemology) that are then examined (methodology, analysis) in specific ways Denzin \& Lincoln [37]. Understanding this difference when determining which approach would be most useful, either by deductively testing safety theories or inductively searching for new theory. In Figure 2, Kyrö [38] depicts how understanding paradigm as a mediator, a gathering link, between philosophical bases and methods, might help in choosing methods and theories for study. 


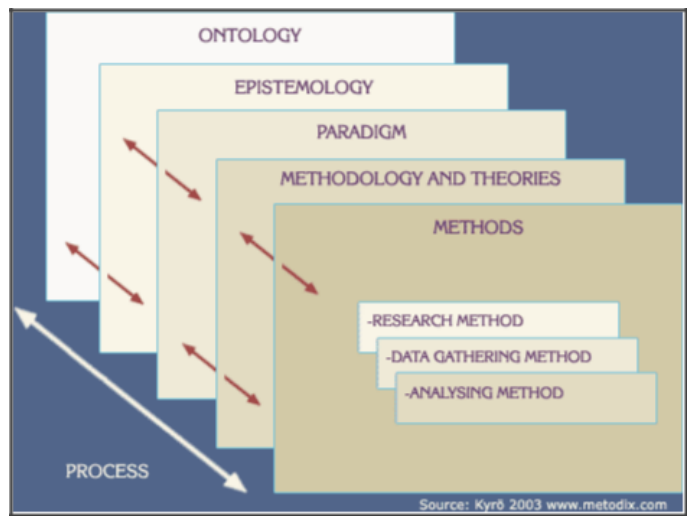

Figure. 2. Hierarchical approach to paradigms [38].

\subsection{Context in Knowledge and Understanding}

Embracing the variety of research paradigms moves beyond the linear trappings of root cause analysis and toward understanding the increasing complexity of modern safety environments. Paradigms are "basic belief system based on ontological, epistemological, and methodological assumptions" [39].

- Ontology: Assumptions about the nature of reality

- Epistemology: How the researcher comes to know that reality

- Methodology: How the researcher accesses and reports what is learned about reality

The directional relationship between these philosophical assumptions can be seen in the work of [40], Figure 3.

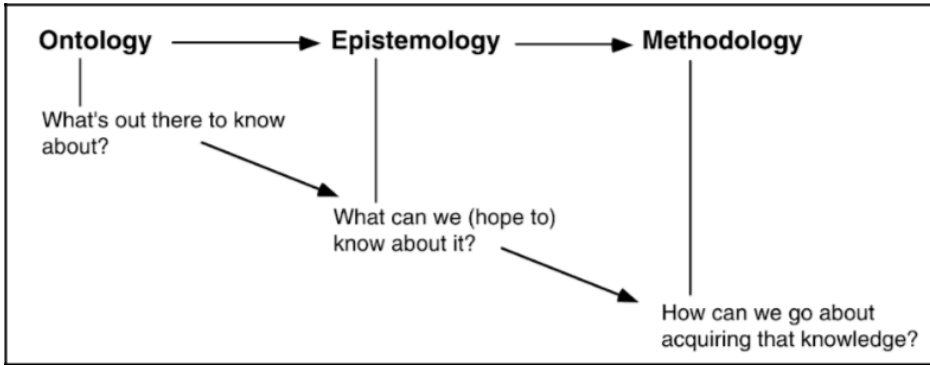

Figure. 3. Ontology, epistemology, methodology; directional dependence [40].

A recent web search revealed as many as 16 paradigms currently in use, although two remain dominant in safety research, Positivist, Interpretivist/Constructivist [41-46]. There is also a growing community of researchers, under the critical realist paradigm, that argue "scientific knowledge does not consist of 'propositions' or 'statements' about events or phenomena; rather, it is comprised of (provisional and fallible) descriptions of structures and powers" [47]. A brief review of the origins of research paradigms follows.

The word "paradigm" stems from late 15th century, Late Latin paradigma and Greek paradeigma meaning a "pattern, model; precedent, example" [14]. In scientific communities, American physicist, historian, and philosopher, Thomas Kuhn's [48] critical work The Structure of Scientific Revolutions, described paradigms as "universally recognized scientific achievements that for a time provide model problems and solutions to a community of 
practitioners." Yet, it was the publication of Ludwik Fleck's [49] Genesis and Development of a Scientific Fact, that introduced "thought collectives" which enlighten Kuhn's definition of paradigms and work on scientific communities of knowledge. "When we look at the formal aspect of scientific activities, we cannot fail to recognize their social structure" [49]. The social scientists' contribution to safety science must be acknowledged as it has increased our understanding of human, organizational, and socio-technical factors influencing safety.

Building on the work of Kuhn and Fleck, Guba and Lincoln [50] define paradigm as "the basic belief system or worldview that guides the investigator, not only in choice of method but in ontologically and epistemologically fundamental ways." When considering research in safety science the paradigm encapsulates the underlying logic and theoretical assumptions that support selection of study methodology. Without a paradigm. Kuhn [48] warned, that the "reason for seeking some form of more recondite information, early fact-gathering is usually restricted to the wealth of data that lie ready to hand."

Data is seldom readily available in qualitative studies, nor easily collected, because the researchers seek understanding in the depth and richness of human experience. Understanding the world, and humans in it, leads to the next section an overview of preeminent philosophers associated with the foundational ontological and epistemological differences in safety science.

\subsection{Preeminent Philosophers}

\subsubsection{The Ontological Divide}

The ontological divide originates in Plato's Protagoras (490-420 B.C.) where Socrates engages in discussion with one of the Sophists, Protagoras. Plato establishes the relativist position through two famous quotes by reference to Protagoras' book:

- "One can only think what he experiences, and what he experiences is true." [51]

- "Of all things the measure is Man, of things that are, that they are, of things that are not, that they are not" [52].

Plato (427 - 347 B.C.), drawing on his experience as a student of Socrates (469-399 B.C.), and the work of Heraclitus (530-470 B.C.) and Parmenides (515-450 B.C.), developed a different philosophy of reality. Plato's view, in contrast with Socrates, was that humans were fallible, limited in our abilities, our senses were inadequate to interpret reality, which he described as Form, or the perfect idea, existing only in the spiritual world [53]. These ontological positions are seen in safety science today as Interpretivism and Positivism respectively.

\subsection{Epistemology divide}

Later, Aristotle's Metaphysics, Books I-XIV, [54], introduced "the first principle of all things" and sets the course of inquiry into What is a thing? Here Aristotle diverged from Plato's view of reality as Forms, existing outside the physical world. Aristotle, more concerned with practical wisdom [Greek, phronesis] argued the world exists in observable physical arrangement "what is actually there... the essence of the thing" but the only way humans experience the world is through our senses, giving humans contact and, at the same time, insulating us from the world [55]. The disagreement between Plato and Aristotle on the meaning of knowledge is relevant still today. 


\subsubsection{Objectivism-Positivism takes center stage - Galileo}

Nearly 2000 years after Aristotle, Galileo (1564-1642), known as The Father of Modern Science, "rejected metaphysics in favor of a secure method of generating knowledge" [56]. In Siderius Nuncius [The Starry Messenger] Galileo (1610) made systematic observations of the Moon, stars, and moons of Jupiter. His methods stood in contrast to Aristotle and the Catholic Church's prevailing geocentric ideology Jakobsen [57]. Galileo's view of the world as objective, existing outside of human reality, as an observable existence to be studied, contributed to the growth and dominance of the positivist paradigm. His view was supported by Descartes, Hobbes, and Leibniz emphasizing the world as elements and calculations Palmer [58]. Over time positivism has changed but the belief in one objective truth remains.

\subsubsection{Postpositivism}

Postpostivism, born from the work Popper's [59] theory of falsifiability, employs similar research methods but accepts that bias cannot be fully removed. "No matter how faithfully the scientist adheres to scientific method research, research outcomes are neither totally objective, nor unquestionably certain" [60]. Truth, for the postpositivist is no longer objective but approached with a measure of skepticism where reality exists outside the human experience, but we are limited in our ability to acquire it. Constructivist, which may include postpositivist, find reality to be a subjective construct shaped by social influence within the human mind. What holds in safety is that both objective and subjective description of events are valuable.

\subsubsection{Interpretivism-Constructivism}

The last of the distinctions of significance in qualitative research in safety is the introduction of constructivism as a paradigm closely related to interpretivism. The two are frequently used interchangeably. For the interpretivist, truth and knowledge are not external to human experience, and "social meaning is not automatically located within an object or situation, it is created by human beings" [61]. As mentioned previously, meaning is the connection between context and creating knowledge, and understanding. It is the creation of meaning that leads to constructivist theory.

Jean Piaget (1896-1980), Swiss biologist and psychologist, along with Lev Vygotsky (1896-1934), Heinz von Foerster (1911-2002), and John Dewey (1859-1952), are recognized as the fathers of constructivism. Their combined contribution has reshaped the epistemological framework that supports continued use of qualitative research and its expansion into safety science. Constructivist theory "defines knowledge as temporary, developmental, socially and culturally mediated, and thus, non-objective" [62]. Piaget posited that "humans cannot be given information, which they immediately understand and use; instead humans must construct their own knowledge" [27]. The interpretivist/constructivist view of the world accepts multiple realities and therefore understands how many people experiencing the same event may all see and remember it differently. Rather than being a detractor, this broadens the context of safety events allowing for further exploration and greater understanding.

\subsubsection{Paradigms are not permanent}

Paradigms provide a "general philosophical orientation to research and can be combined or used individually" [41]. The type of research questions developed in safety studies lead the researcher toward a specific theoretical approach. In the qualitative heuristic approach, Abbott [63] argued "the idea of heuristics is to open new topics, to find new things. To do 
that, sometimes we need to invoke constructivism. Sometimes we need a little realism." Additionally, paradigms are constructed through social behavior, therefore "fluid and how we think or behave cannot be completely compartmentalized with clear-cut boundaries. As such, to think about a paradigm as a worldview or epistemological stance does not preclude the cross-over of ideas" [44]. The ability to adapt and adjust paradigms is one of the benefits of qualitative inquiry allowing researchers to explore phenomenon from many angles.

\section{Qualitative Inquiry Benefits and Case Examples}

\subsection{Benefits}

There are several benefits associated with qualitative research in safety science, key among them are, understanding individual and organizational behavior, predictive and emergent theories, flexibility, and acceptance of multiple truths. There are also some disadvantages, such as being highly subjective, finding is not easily graphed and should be tailored for decision makers, and data is not intended to be replicated. Nonetheless, qualitative inquiry is an excellent tool used independently or in conjunction with quantitative, critical realism, or mixed method studies.

Helman [64] reported that qualitative methodologies "provides the most useful means of understanding why people behave in certain ways and for exploring the relationship between beliefs and behavior." Unlike other methods, qualitative research, using open-ended questions, is uniquely positioned to gather narrative data providing deep and rich information about the environment and conditions surrounding safety work. Hummerdal [35] addresses the importance of capturing stories that "draw attention to things that are entangled and emergent." His use of the term "messy details" describes the complex safety environment in modern socio-technical systems. "It brings out the messy details and other real aspects that impact how work is carried out. Relationships, feelings, mess, smells, perspiration, sounds, screaming, anxiety, pain, joy, pride and camaraderie are all highly unlikely to be captured by big data. Yet, all these aspects impact work, and safety" [35]. Qualitative research also reveals critical safety information or what Love, Smith, Ackermann, \& Irani [65] refer to as "uncomfortable knowledge and inconvenient truths that can confront projects in everyday practice""

Another key feature of qualitative research is the focus on developing predictive or emerging theory. Charmaz [66] notes that "qualitative researchers have one great advantage over our quantitative colleagues. We can add new pieces to the research puzzle or conjure entire new puzzles - while we gather data - and that can even occur late in the analysis. The flexibility of qualitative research permits you to follow leads that emerge." While frequently associated with the grounded theory method, emergence may be found in any interpretive study, especially those employing open-ended questions. "By creating open-ended, nonjudgmental questions, you encourage unanticipated statements and stories to emerge" [66] that often reveal latent or unknown safety threats.

Through qualitative data collection and analysis multiple truths may emerge. "Researchers conducting qualitative studies embrace the ontological assumption of multiple truths or multiple realities, i.e., that each person has an understanding of reality from an individual perspective" [67]. Each individual shapes memory and meaning independently, no two experiences are identical. The qualitative researcher's role is not to ascertain which account is the more accurate or 'truthful,' or who is more 'believable.' Instead, to understand how 'situated accounts' are told in a way that allows the speaker to achieve a different purpose through emphasizing some aspect of their stories and deemphasizing others [68]. 


\subsection{Examples of Qualitative Benefits in Completed Studies}

Three recent examples are included to provide the reader with insight into the benefits of qualitative inquiry in safety. These examples demonstratives one or more of the benefits of qualitative research. Note the flexibility, adaptability, and emergent theories within these safety studies.

\subsubsection{Finnish Maritime}

A Finnish maritime safety, conducted by Teperi, Lappalainen, Puro, and Perttula [69] offers a good example of the emergent property of qualitative research. Amongst the many findings revealed from analysis of data collected from " 17 operative employees and 12 safety and unit managers" was that inspections conducted by maritime authorities focus almost exclusively on technical matters with little attention paid to psychosocial factors and Human Factors. These types of emergent factors are valuable to organizations, and regulators, in developing effective safety policies and practices, including inspection reports.

This study revealed that "maritime safety culture has not yet proceeded from Safety-I thinking to Safety-II." During the study, the authors explained that several participants defined safety reporting as weak. One of the participants is quoted saying "that (reporting incidents) has been quite weak. If something really serious happens, only then we report, but otherwise it's quite sparse." The maritime study deftly brings to the surface participants individual experience, their situated understanding of events, and contributes a great deal to the larger picture of safety. This type of context is essential to developing organizational or industry-wide safety intelligence.

\subsubsection{Australian Mining}

Equally important, and a strength of qualitative research, is when successful measures and practices emerge from the research. Such was the case in Havinga, Dekker, and Rae's [70] study of blast crews within an Australian mining operation. One aim of their "study was to investigate the possibilities of crew resource management training for blast crews in mining." To the researchers' surprise, what they found was "practitioners purposely left 'traces' for themselves and other crewmembers in the environment" and "a lot of communication was happening nonverbally, although not necessarily implicit " As reminders, member of the blast crew would leave stones to alert other team members to hazards, along with other forms of non-verbal communication. The actions of the blast crew were not codified in procedures or policy but developed as team norms. In this study flexibility and emergent aspect of qualitative research contributed to broadening context and heightened understanding, which in turn served to enhance organizational safety by increasing adaptive capacity and resilience.

\subsubsection{Aviation}

Lastly, I offer an example from aviation through Lai, Chen, Khoo, \& Zheng [71] qualitative study of unstable approaches to landing. "Executing a successful landing requires meeting all the stable approach criteria, including proper airspeed, altitude, power setting, etc. Otherwise, an unstable approach can occur." They examined shared mental models of unstable approaches using critical decision method to conduct semi-structured interviews of nine airline pilots and seven air traffic controllers. The significance of this qualitative study is apparent considering "the safety data from the IATA GADM Accident database show that the approach and landing phases of flight account for the major proportion of all commercial aircraft accidents; $65 \%$ of the total accidents recorded from 2011-2015. Unstable approaches were identified as a factor in $14 \%$ of those accidents" [72]. 
The researchers explored how informational, operational, evaluative, and team-related mental model disconnects, occur between pilots and air traffic controllers that results in inconsistent shared mental models and increased risk of unstable approaches. Six mental model disconnects for pilots and five for air traffic controllers were identified. I cannot cover the paper in detail, and leave that to the reader to pursue, but include one prominent conclusion that highlights how qualitative research increases context in safety studies. "Based on the results obtained, the unequal information sharing is the major factor which causes task-related operational disconnects...Therefore, the improvements in information sharing in the landing system deserve more attention" [71].

Through the narrative accounts of these pilots and air traffic controllers, and with the researchers' openness to multiple truths, the need for increased key information emerged. Pilots with their limited view of the airspace environment seek specific targeted information to shape their understanding, and mental model, to more closely align with the controllers. Together they capture the context of the airspace and aircraft management to ensure safety.

\section{Conclusion}

Expanding the contextual lens in safety science is about broadening our understanding of the environment and conditions people negotiate in normal day-to-day operations. While all methods of research have a role to play qualitative research is uniquely suited to capture and analyze stories from those with first-hand experience of how work-is-done, and how it differs from work-is-imagined. It is through their individual experience, when analyzed collectively, that a greater context emerges allowing for increased understanding and action.

As revealed in the three recent examples, the researchers' flexibility and willingness to accept multiple truths, coupled with non-judgmental open-ended questions creates a pathway to new knowledge and understanding that builds safety intelligence. There is little question it is time for greater emphasis on using qualitative research, both independently and in conjunction with quantitative studies in today's complex and dynamic safety environment. The time is right to explore new ways of not only looking at safety sensitive work but also listening to those with the immediate experience. Qualitative research in safety science brings the diversity of experience into a collective understanding that supports strong safety culture.

\section{References}

1. E. Hollnagel, Safety Sci._67, 21 (2014)

2. H. Bhogle. Indian Expr News. (2011).

3. C.V. Ferroff, T.J. Mavin, P. R. Bates, P. S. Murray, Aeronautica, 2, 1 (2012)

4. S. Kaspers, N. Karanikas, S. Piric, R. van Aalst, R.J. de Boer, A. Roelen, PESARO 2017. The 7thIARIA, 9 (2017)

5. K.V. Størkersen, Antonsen, T. Kongsvik, J. Risk Research, 20, 1154, 1168 (2017)

6. P.B. Checkland, Human Sys. Mgmt, 8, 279 (1989)

7. K. Yilmaz, K. Eur. J Ed, 48, 313 (2013)

8. P. Halfpenny, Soc. Review, 27, 799 (1979)

9. W. Hong, K.R. Lang, A Philosophical Foundation of Qualitative Modeling Methodologies Based on the Yin-Yang Principle. 134 (2001).

10. J, Carrier, Cardiff Sch. of Health. Sci. 8 (2018)

11. M. Bazire, P. Brézillon, CONTEXT 2005, 31 (2005)

12. T.A. Van Dijk, Discourse and Cont. Soc. Chng. 54, 285 (2007)

13. J.S. Brown, P. Duguid, The Social Life of Information: Updated, with a New Preface, 100 (2017)

14. context, paradigm. Etymonline. (2019) 
15. T. Greenhalgh, G. Robert, P. Bate, F. Macfarlane, O. Kyriakidou, Diffusion of innovations in health service organisations: a systematic literature review. 195 (2008)

16. R. Robinson, Urban Tech: People, Place, Tech. (2015)

17. G. Wong, Safetydifferently.com. (2018)

18. A. Duranti, C. Goodwin, Rethinking context: Language as an interactive phenomenon 11, 4 (1992)

19. P. Bate, Perspectives on Context. 8 (2018)

20. D. Hummerdal, Safetydifferently.com. (2018)

21. M. Dixon-Woods, Perspectives on Context. 95 (2014)

22. A. Oswalt, M. Dombeck, Rethinking context: Language as an interactive phenomenon. 11, 300 (2005)

23. A.C. Edmondson, Div. of Research, Harvard Bus. 255 (2002)

24. A.K. Cebrowski, J.W. Raymond, Operationally responsive space: A new defense business model. 77 (2005)

25. L. Resnick, Knowing, learning, and instruction: Essays in honor of Robert Glaser. 2 (2016)

26. E. von Glasersfeld, The invented reality, 17. (1984)

27. J. Piaget, To understand is to invent: The future of education. 53 (1973)

28. D. Perkins, The Education Digest. 59, 39 (1998)

29. T. Conklin, Pre-Accident Podcast. (2019)

30. S. Dekker, Safety differently: human factors for a new era. (2014)

31. E. Hollnagel, Safety-I and safety-II: the past and future of safety management. (2018)

32. D.D. Woods, E. Hollnagel, Resilience engineering. 15 (2017)

33. S. Dekker, HindSight, EUROCONTROL, 25, 8 (2017)

34. E. Hollnagel, R.L. Wears, J. Braithwaite, From Safety-I to Safety-II: a white paper. 23 (2015)

35. D. Hummerdal, Safetydifferently.com (2016)

36. T. Conklin, Pre-Accident Investigations: Better Questions-An Applied Approach to Operational Learning. 42 (2016)

37. N.K. Denzin, Y.S. Lincoln, The landscape of qualitative research, 1, 23 (2008)

38. P. Kyrö, Metodix. (2002)

39. E.G. Guba, Y.S. Lincoln, Naturalistic inquiry. 107 (1985)

40. C. Hay, Political analysis: a critical introduction. 64 (2002)

41. J. W. Creswell, J. D. Creswell, Research design: Qualitative, quantitative, and mixed methods approaches. (2017)

42. B. Chilisa, Indigenous research methodologies. (2011)

43. J. Jericho, The Free School. (2017)

44. C. Kivunja, A. B. Kuyini, Int. J. Higher Ed. 6, 26 (2017)

45. Y.S. Lincoln, S.A. Lynham, E.G. Guba, The Sage handbook of qualitative research, 4, 97 (2011)

46. D.M. Mertens, Int. J. of Multiple Research App., 4, 9 (2010).

47. P.S. Gorski, What is critical realism? And why should you care? 669 (2013)

48. T.S. Kuhn, The structure of scientific revolutions. 15 (1962/2012)

49. L. Fleck, Genesis and development of a scientific fact. $42(1935 / 2012)$

50. E.G. Guba, Handbook of Qualitative Research. 11105 (1994)

51. G.J. Mattey, UC Davis Philosophy 21. (2001)

52. D.W. Graham, The texts of early Greek philosophy: the complete fragments and selected testimonies of the major Presocratics. 703 (2010)

53. L. Slawson, Owlcation. (2018)

54. N. Ross, Metaphysics. 73 (2015) 
55. B. Gore, Cosmo Learning. (2011)

56. J.C. Pitt. Galileo, human knowledge, and the book of nature: Method replaces metaphysics 50, 4 (2013)

57. T.G. Jakobsen, Popular Soc. Sci. (2013)

58. D. Palmer. Looking at philosophy: The unbearable heaviness of philosophy made lighter. (2009)

59. K. Popper, The logic of scientific discovery. (1934/2005)

60. M. Crotty, The foundations of social research: Meaning and perspective in the research process. (1998)

61. S. Dyson, B. Brown, Social theory and applied health research. 19 (2005)

62. J. Brooks, M. Brooks. In Search of Understanding: The Case for Constructivist Classrooms. vii (1993)

63. A. Abbott, Methods of discovery: Heuristics for the social sciences. 191 (2004)

64. C.G. Helman, Primary care research: Traditional and innovative approaches, 105 (1991)

65. P. Love, J. Smith, F. Ackermann, C. Irani, Int. J. of Project Mgmt. (2019)

66. K. Charmaz, Constructing grounded theory: A practical guide through qualitative analysis.14 (2006)

67. C. Erlingsson, P. Brysiewicz, African J. of Emergency Med, 3, 92 (2013)

68. R. Barbour, Introducing qualitative research: a student's guide. (2013)

69. A.M. Teperi, J. Lappalainen, V. Puro, P. Perttula, WMU J of Maritime Affairs, 17 (2018).

70. J. Havinga, S. Dekker, A. Rae, Theo. Issues in Erg. Sci, 19, 213 (2018).

71. H.Y. Lai, C.H. Chen, L.P. Khoo, P. Zheng, Rel. Eng. and Sys. Safety, 185, 383 (2019).

72. International Air Transport Association (IATA). $2^{\text {nd }}, 49$ (2016) 